\title{
Exkurs: Ist der Verein noch eine zeitgemäße Rechtsform für sozialwirtschaftliche Unternehmen?
}

Im Zusammenhang mit der zunehmenden wirtschaftlichen Betätigung einiger sozialkaritativer Vereine ist auf ein aktuelles Urteil zur Haftung von Vereinen und Vereinsmitgliedern des Bundesgerichtshofs für Zivilsachen hinzuweisen, in dem er ein aufsehenerregendes Urteil des Oberlandesgericht Dresden inhaltlich nicht bestätigt hat.

Die Dresdener Richter hatten mit Urteil vom 9. August 2005 für den Fall eines in der Insolvenz befindlichen Vereins eine Durchgriffshaftung der Mitglieder des Vereines angenommen. Das Gericht hatte seiner Entscheidung die Wertung zugrunde gelegt, dass Mitglieder eines personalistisch strukturierten eingetragenen Vereins, der sich über das sogenannte Nebenzweckprivileg hinaus wirtschaftlich betätige, wegen Missbrauchs der Rechtsform akzessorisch für die Vereinsverbindlichkeiten haften könnten. Dieses gelte zumindest dann, wenn die Mitglieder Kenntnis von der wirtschaftlichen Betätigung des Vereines hätten und dieser keinen Einhalt geböten. Vor dem Hintergrund dieser Entscheidung wurde zunehmend in Frage gestellt, ob die Rechtsform des eingetragenen Vereines insbesondere für das Betreiben von steuerbegünstigten Sozialunternehmen weiterhin als geeignete und zukunftsfähige Gesellschaftsform angesehen werden könne oder ob angesichts bestehender Haftungsrisiken andere Rechtsformen anzuraten seien.

Mit Urteil vom 10. Dezember 2007 hat nun der Bundesgerichtshof entschieden, dass im Falle des insolventen Vereines keine Durchgriffshaftung der Mitglieder anzunehmen ist (Az. II ZR 239/05). Das Gericht hat in seinem Urteil ausgeführt, dass die vom Oberlandesgericht Dresden angenommene akzessorische Haftung der Mitglieder des Vereines für dessen Verbindlichkeiten im Wege einer Durchgriffshaftung wegen Rechtsformmissbrauchs mit dem geltenden Gesetzesrecht nicht in Einklang stehe. Es entspräche insofern ständiger höchstrichterlicher Rechtsprechung, dass für Verbindlichkeiten eines eingetragenen Vereins grundsätzlich nur dieser selbst und nicht die hinter ihm stehenden Vereinsmitglieder haften würden. Eine Durchbrechung dieses Trennungsgrundsatzes sei nur ausnahmsweise dann zulässig, wenn die Ausnutzung der rechtlichen Verschiedenheit zwischen der juristischen Person und den hinter ihr stehenden natürlichen Personen rechtsmissbräuchlich sei. Da das Vorliegen eines derartigen Rechtsmissbrauchs vorliegend nicht festzustellen sei, sei eine Durchgriffshaftung nicht geboten. Zwar sei das Überschreiten des Nebenzweckprivilegs nicht zu rechtfertigen. Für derartige Verstöße sehe das Gesetz jedoch gegebenenfalls ein Amtslöschungsverfahren nach den §§ 159, 142 FGG oder die Entziehung der Rechtsfähigkeit nach § 43 Abs. 2 BGB als Sanktion für den Verein vor. Eine Durchgriffshaftung der Mitglieder sei hingegen nicht gerechtfertigt. Aufgrund dessen hat der Bundesgerichtshof das Urteil des Oberlandesgericht Dresden in dieser Hinsicht aufgehoben.

Der Bundesgerichtshof hat somit sehr deutlich die bisherige Maxime der Rechtsprechung bestätigt, dass eine Durchgriffshaftung von Vereinsmitgliedern grundsätzlich nur in wenigen Ausnahmefällen in Betracht kommt. Festzuhalten ist daher, dass zumindest zur Abwendung von Haftungsrisiken ein Rechtsformwechsel oder die Wahl anderer Rechtsformen als die des Vereines nicht erforderlich erscheint. Grundsätzlich stellt somit auch der eingetragene Verein weiterhin eine geeignete Rechtsform zum Betreiben steuerbegünstigter Sozialunternehmen dar, wobei jedoch die seitens des Gerichtes angesprochenen weiter möglichen Sanktionen nicht außer Acht gelassen werden sollten.

Stephanie Krekeler

\section{Arbeitshilfe: Corporate Governance sozialtätiger Unternehmen}

Die Solidaris Unternehmensberatungs-GmbH hat eine 128 Seiten umfassende Broschüre vorgelegt: »Corporate Governance sozialtätiger Unternehmen - Grundlagen und Hinweise für Verantwortliche in Geschäftsführungs- und Aufsichtsgremien«. Die Schrift vermittelt grundlegende Informationen zur rechtlichen und faktischen Gestaltung der Zusammenarbeit der Leitungsorgane in Unternehmen des Gesundheitsund Sozialwesens. Auf Basis einer ausführlichen Bestimmung des Begriffs »Corporate Governance« werden die einschlägigen gesetzlichen Vorgaben sowie die von den verfassten Kirchen und den Wohlfahrtsverbänden hierzu herausgegebenen Empfehlungen, Kodices und Ansätze zur Gestaltung der Corporate Governance vorgestellt. Da die Gesetze und Empfehlungen zur Gestaltung der Corporate Governance ausnahmslos ein duales Führungssystem aus Geschäftsführungsund Aufsichtsorgan empfehlen, werden die Rahmen- bedingungen dieser Führungsstruktur im Hinblick auf unterschiedliche Rechtsformen $(\mathrm{GmbH}$, Verein, Stiftung, Körperschaft des öffentlichen Rechts) erörtert. Daran anschließend gibt die Arbeitshilfe zahlreiche Hinweise zur Ausgestaltung der Zusammenarbeit der Leitungsorgane in sozialtätigen Unternehmen, ergänzt um Ausführungen zu deren Kontrolle, die unter anderem von externen Instanzen (z. B. Aufsichtsbehörden, Abschlussprüfer) ausgeübt wird. In einem gesonderten Abschnitt gibt die Arbeitshilfe abschließend Hinweise zur Haftung der Mitglieder von Geschäftsführungsund Aufsichtsorganen. Der Anhang enthält eine umfangreiche »Checkliste zur unternehmensspezifischen Gestaltung der Corporate Governance«. Die Arbeitshilfe kostet 11,- Euro zuzüglich Versandkosten.

E-Mail bestellung@solidaris.de oder Fax 02203 8997-197 